group required ICU admission vs $16.32 \%$ and $13.98 \%$ required ventilation vs $6.9 \%, p<0.001$. The average age was higher in the arthropathy arm vs control at 66.56 years old vs $51.53, p<0.001$. Charlson comorbidity index was also higher in the arthropathy arm at 2.72 vs $0.96, p<0.001$.

Conclusion: This is a large analysis of inflammatory arthropathy patients hospitalized with COVID-19. While the arthropathy group was older, and had more co-morbidities, when adjusting for potential confounders, inflammatory arthropathy patients had a higher risk of death and mechanical ventilation, as well as longer length of stay.

Disclosure of Interests: None declared.

DOI: 10.1136/annrheumdis-2021-eular.482

\section{POS0048 SEROPREVALENCE OF ANTI-SARS-COV-2 ANTIBODIES IN RHEUMATIC PATIENTS TREATED WITH BIOLOGICAL AND TARGETED THERAPY LIVING IN LOMBARDY, ITALY (MAINSTREAM PROJECT)}

E. G. Favalli', G. Maioli ${ }^{1}$, M. Bombaci ${ }^{2}$, M. Biggioggero ${ }^{1}$, A. Favalli ${ }^{2}$, E. Agape ${ }^{1}$, G. Andrea ${ }^{2}$, E. Pesce' ${ }^{2}$, E. Zagato ${ }^{2}$, T. Fabbris' ${ }^{2}$ M. Martinovic ${ }^{2}$, E. Marchisio ${ }^{3}$, S. Abrignani ${ }^{2,4}$, R. Grifantini ${ }^{2}$, R. Caporali ${ }^{1,5},{ }^{1}$ ASST Gaetano Pini-CTO Institute, Division of Clinical Rheumatology, Milan, Italy; ${ }^{2}$ Istituto Nazionale Genetica Molecolare, Padiglione Romeo ed Enrica Invernizzi, Milan, Italy; ${ }^{3}$ Dia.Pro, Diagnostic Bioprobes srl, Milan, Italy; ${ }^{4}$ Università degli Studi di Milano, Department of Clinical Sciences and Community Health, Milan, Italy; ${ }^{5}$ Università degli Studi di Milano, Department of Clinical Sciences and Community Health, Research Center for Adult and Pediatric Rheumatic Diseases, Milan, Italy

Background: Emerging observational data have shown that rheumatic patients seem not to be more susceptible to SARS-CoV-2 infection neither to worse outcomes. However, the true prevalence of COVID19 is still unknown due to the high proportion of subclinical infection. In this scenario, measuring the seroprevalence of SARS-CoV-2 may be crucial to improve the knowledge about the impact of COVID19 in rheumatic patients.

Objectives: To estimate in a COVID19 high-endemic area (Lombardy, Italy) the prevalence of anti-SARS-CoV-2 antibodies in a large cohort of patients with rheumatoid arthritis (RA) or spondyloarthritis $(\mathrm{SpA})$ treated with biologic (b-) or targeted synthetic (ts-) disease modifying drugs (DMARDs).

Methods: A seroprevalence cross-sectional study was conducted in the period between $4^{\text {th }}$ May and $16^{\text {th }}$ June 2020, including patients with confirmed RA or $\mathrm{SpA}$ treated with b- or tsDMARDs. Patients were tested for anti-SARS-CoV-2 $\operatorname{lgG}$, IgM and IgA antibodies against main viral antigens (nucleoprotein [N], spike 1 [S1], receptor-binding domain [RBD]) using ELISA. These data were compared with those observed in the healthy population in the same period and region. Patients also answered a questionnaire on history of symptoms consistent with COVID19, risk factors and comorbidities. Serological response to RBD was evaluated according to symptom severity (asymptomatic, minor, or major [respiratory and fever $>37.5^{\circ} \mathrm{C}$ ] symptoms).

Results: The study population included 300 patients $(62 \%$ females, mean age 53 years, $20 \%$ over 65 years old) diagnosed with RA (56\%), psoriatic arthritis $(23 \%)$, or ankylosing spondylitis $(21 \%)$, treated with anti-TNF $(57 \%)$, abatacept $(20 \%)$, anti-IL6 $(11 \%)$, or JAK inhibitors $(5 \%)$. Four patients $(1.3 \%)$ referred a prior diagnosis of COVID19 defined by nasopharyngeal swab. Immunoglobulin titers were evaluated resulting in $9 \%, 13.6 \%$, and $13.3 \%$ positive patients for $\lg G$, IgM and IgA, respectively (Table 1), with no significant difference to the healthy population. Among seropositive patients, $55.3 \%$ were asymptomatic, $16 \%$ had minor and $19.6 \%$ major symptoms, $7.1 \%$ were hospitalized. No deaths or admission to intensive care units occurred. IgM, IgG and IgA titers to RBD were higher in patients with both minor and major symptoms compared with asymptomatic ones (Figure 1). No differences were found between seronegative and seropositive patients in relation to age, sex, rheumatic diagnosis, and treatments with b- or tsDMARDs. A relative lower risk of seropositivity was observed in patients receiving concomitant methotrexate (RR $0.49,95 \% \mathrm{Cl} 0.25-0.94 ; \mathrm{p} 0.04$ ), while an increased risk was associated with obesity (RR 2.33,95\% Cl 1.26-3.79; p 0.019) and presence of at least 2 comorbidities (RR 1.94, 95\% Cl 1.11-3.15; p 0.037). Corticosteroids use was numerically more frequent in seropositive than seronegative patients (18\% vs $14 \%$ ).

Conclusion: This study confirms that, even in a cohort of rheumatic patients, the spread of SARS-CoV-2 infection is much greater than that observed by capturing only swab-diagnosed COVID19 cases. The underlying rheumatic disease and ongoing therapy with b/ts-DMARDs do not seem to impact SARS-CoV-2 antibody positivity, which conversely seems to be proportional to the intensity of COVID19 symptoms and less frequent in patients receiving concomitant methotrexate. The project was co-financed by Lombardy Region 2014-2020 Regional Operational Programme under the European Regional Development Fund.
Table 1. Prevalence of specific anti-SARS-CoV-2 antibodies.

\begin{tabular}{lccc}
\hline Antibodies & Posivite(n) & Seroprevalence $(\%)$ & $(95 \% \mathrm{Cl})$ \\
\hline $\operatorname{IgG}$ & 27 & $9 \%$ & $(6.2-12.7)$ \\
IgG anti-N & 26 & $8.6 \%$ & $(5.9-12.3)$ \\
IgG anti-RBD & 20 & $6.6 \%$ & $(4.3-10)$ \\
IgG anti-S1 & 18 & $6 \%$ & $(3.8-9.2)$ \\
IgM & 41 & $13.6 \%$ & $(10.2-18)$ \\
IgM anti-N & 35 & $11.6 \%$ & $(8.5-15.7)$ \\
IgM anti-RBD & 25 & $8.3 \%$ & $(5.7-12)$ \\
IgA & 40 & $13.3 \%$ & $(9.9-17.6)$ \\
IgA anti-N & 37 & $12.3 \%$ & $(9.0-16.5)$ \\
IgA anti-RBD & 25 & $8.3 \%$ & $(5.7-12)$ \\
IgG+IgM & 23 & $7.6 \%$ & $(5.1-11.2)$ \\
IgG+IgM+IgA & 22 & $7.3 \%$ & $(4.9-10.5)$ \\
IgG+IgA & 24 & $8 \%$ & $(5.4-11.6)$ \\
IgG/IgM/IgA & 56 & $18.6 \%$ & $(14.6-23.4)$ \\
\hline
\end{tabular}
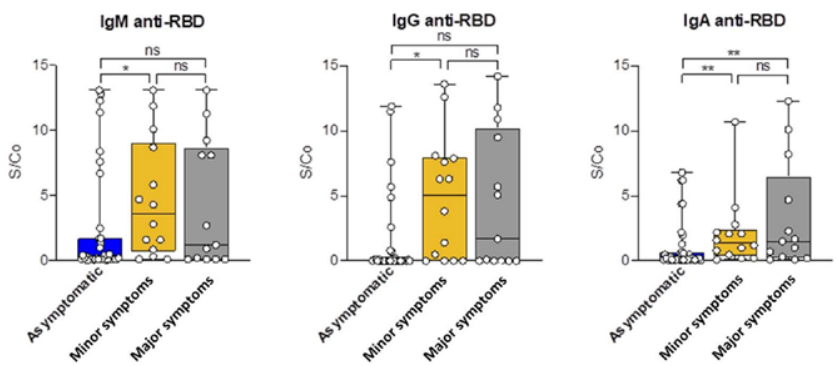

Figure 1. Antibody levels (S/Co) against SARS-CoV-2 RBD.

Disclosure of Interests: None declared.

DOI: 10.1136/annrheumdis-2021-eular.594

\section{POS0049 \\ SARS-COV-2 INFECTION IN CHILDREN WITH RHEUMATIC DISEASE: EXPERIENCE OF A TERTIARY REFERRAL CENTER}

B. Sözeri ${ }^{1}$, F. Demir ${ }^{1}$, S. Kalın ${ }^{2}$, C. Hasbal Akkuş ${ }^{3} .{ }^{1}$ İstanbul Health Sciences University, Umraniye Training and Research Hospital, Pediatric Rheumatology, Istanbul, Turkey; ${ }^{2}$ İstanbul Health Sciences University, Umraniye Training and Research Hospital, Pediatric Radiology, Istanbul, Turkey; ${ }^{3}$ Istanbul Health Sciences University, Umraniye Training and Research Hospital, Pediatrics, Istanbul, Turkey

Background: Patients with rheumatic diseases are considered at risk for serious infections due to their immune-compromised-status set in their primary systemic disease and the usage of immune-modulating therapies. Although various results have been reported on the subject, it is still unknown whether patients with rheumatic disease, many of whom are on immune-suppression, are at higher risk of severe COVID-19.

Objectives: We aim to share our clinical SARS-CoV-2 experience in patient with the childhood rheumatic disease during pandemic.

Methods: We evaluated 4470 patients at our pediatric-rheumatology clinic during the pandemic, from 11-March to 15-October-2020. Demographic and clinica features, treatments, laboratory results, imaging findings, and clinical outcomes of patients diagnosed with COVID-19 and/or multisystem inflammatory syndrome (MIS-C) were review from the medical records. The data of all these patients were compared between groups and presented. A p-value $<0.05$ was considered statistically significant.

Results: Among 4470 patients, 87 COVID-19 suspected patients were included in the study. Fifty six (64.4\%) patients were hospitalized and 31 were followed without hospitalization. The most common rheumatic diseases among them were juvenile idiopathic arthritis and familial Mediterranean fever (35.6\% and $34.5 \%)$. The primary disease status of these patients were; 78 $(89.6 \%)$ were in remission, while $9(10.3 \%)$ had active disease at the time of COVID-19 diagnosis. Twenty six of these patients were treated with biologic DMARDs. SARS-CoV-2 infection (RT-PCR and/or antibody test) was found positive in 84 patients $(96.5 \%)$. Also, fifty one $(58.6 \%)$ patients had an epidemiologic contact to a person with COVID-19. Fifty six of $87(64.3 \%)$ had a fever and $20(23 \%)$ had a fever for five or more days. Gastrointestinal system involvement was in $11(12.6 \%)$, the respiratory system was in $40(46 \%)$ and fatigue was in $57(65.5 \%)$ patients. Cutaneous involvement was seen in 5 patients including maculopapular rash in two, vasculitic rash in two, and chilblain in one patient. $63.2 \%$ of patients had increased C-reactive protein (CRP), $40.2 \%$ had lymphocytopenia $(<1500 / \mathrm{mm} 3)$ and $26.4 \%$ had elevated D-dimer 
level. SARS-CoV-2 infection was confirmed in 84 patients $(96.5 \%)$. The diagnosis was confirmed by RT-PCR in 74 patients and by antibody test in 10.18 patients met the clinical criteria and diagnosed with MIS-C. Nine of them had also hypotension and seven patients admitted the intensive care unit because of shock and severe end-organ illness. COVID-19 outbreak also caused exacerbation of systemic disease in 56 children due to a discontinue of medication, postponed drug switch, or viral infection triggered.

Conclusion: In conclusion, children with rheumatic disease do not appear to present a higher risk of severe COVID-19. Whether these patients receive biological treatment does not affect the severity of the disease, but it is still not true to say that these drugs are protective. The immunosuppressive treatments can be adjusted in case of infection, otherwise it is not recommended interrupt the treatments. Physicians should be cautious about the hyperinflammatory syndrome associated with COVID-19 in rheumatic children, which may be severe in this group of patients and may be confused with primary diseases.

\section{REFERENCES:}

[1] Wahezi DM, Lo MS, TB Rubinstein, Ringold S, Ardoin SP, Downes KJ, et al. American College of Rheumatology Guidance for the Management of Children with Pediatric Rheumatic Disease During the COVID-19 Pandemic: Version 1. Arth \& Rheum 2020;doi:10.1002/art.41455.

Disclosure of Interests: None declared.

DOI: 10.1136/annrheumdis-2021-eular.1164

\section{POS0050 ARE PATIENTS WITH IMMUNE MEDIATED INFLAMMATORY DISEASES (IMID) MORE LIKELY TO RECEIVE COVID-19 TESTS AND TEST POSITIVE FOR SARS-COV-2? A MATCHED POPULATION-BASED STUDY}

L. Eder ${ }^{1,2}$, R. Croxford ${ }^{3}$, A. Drucker ${ }^{2,4}$, A. Mendel ${ }^{5}$, K. Bindee ${ }^{4,6}$, Z. Touma ${ }^{4,7}$, S. Johnson ${ }^{4,7}$, R. Cook $^{8}$, S. Bernatsky ${ }^{5}$, N. Haroon ${ }^{1,7}$, J. Widdifield ${ }^{3,4,9}$ ${ }^{1}$ University of Toronto - St. George Campus, Medicine and Institute of Medical Science, Toronto, Canada; ${ }^{2}$ Women's College Hospital, Medicine, Toronto, Canada; ${ }^{3}$ ICES, NA, Toronto, Canada; ${ }^{4}$ University of Toronto - St. George Campus, Medicine and Institute of Health Policy Management and Evaluation, Toronto, Canada; ${ }^{5}$ McGill University, Medicine, Montréal, Canada; ${ }^{6}$ Sinai Health System, Medicine, Toronto, Canada; ${ }^{7}$ Toronto Western Hospital, Medicine, Toronto, Canada; ${ }^{8}$ University of Waterloo, Biostatistics, Waterloo, Canada; ${ }^{9}$ Sunnybrook Health Sciences Centre, Research Institute, Toronto, Canada

Background: Population-based data about the COVID-19 risk in patients with IMID remain scarce.

Objectives: To investigate the cumulative incidence and risk factors for laboratory-confirmed COVID-19 infection and SARS-CoV2 testing in patients with IMID compared with matched non-IMID patients from the general population.

Methods: A population-based, matched cohort study was conducted using health administrative data from adults living in Ontario, Canada from January to December 2020. Cohorts for each of the following IMID were assembled: rheumatoid arthritis (RA), psoriasis, psoriatic arthritis, ankylosing spondylitis, systemic autoimmune rheumatic diseases (including lupus, systemic sclerosis, Sjogren's, inflammatory myositis), multiple sclerosis (MS), iritis, inflammatory bowel disease (IBD), polymyalgia rheumatica (PMR) and vasculitis. Each patient was matched with 5 non-IMID comparators based on age, sex, area of residence and living in long term care (LTC). Standardized cumulative rates of testing for SARS-CoV2, and for receiving a positive test between IMID and non-IMID were compared between IMID and non-IMID patients. Multivariable logistic regression analyses assessed sociodemographic factors associated with COVID-19 testing and positivity.

Results: A total of 493,499 IMID patients and 2,466,946 non-IMID comparators were assessed. Significantly more IMID patients versus non-IMID were tested for SARS-CoV2 $(27.4 \%$ vs. $22.7 \%)$, while the proportions of those positive for COVID-19 were identical ( $0.9 \%$ of all patients in both groups). Overall, IMID patients were more likely to undergo SARS-CoV2 testing (odds ratio (OR) $1.28,95 \% \mathrm{Cl} 1.27,1.29)$, but their overall risk of laboratory-confirmed COVID-19 was not elevated (OR $0.97(95 \% \mathrm{Cl} 0.93,1))$. However, the risk of laboratory-confirmed COVID-19 infection was lower in IBD (OR 0.75), MS (OR 0.77) and psoriasis (OR 0.94) and marginally higher in RA (OR 1.07) and iritis (OR 1.13) compared with non-IMID comparators (Figure 1A). The highest standardized rates of COVID-19 infection were found in vasculitis (115 per 10,000 patients) and iritis (109 per 10,000 patients) (Figure 1B). Risk factors for COVID-19 infection included younger age, living in LTC, multimorbidity, urban living and lower income (Table 1).

Conclusion: Patients across all IMID were more likely to be tested for COVID19 versus non-IMID patients. IMID patients were not at higher risk for testing positive for COVID-19 as an overall group, yet risk varied across disease subgroups.

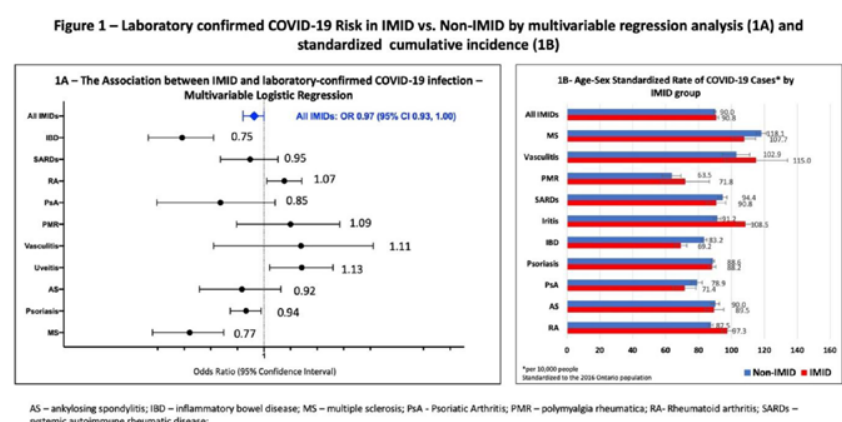

Table 1. Factors associated with COVID-19 infection in IMID vs. Non-IMID - Multivariable Logistic Regression

\begin{tabular}{lcc}
\hline Variable & OR & Cl 95\% \\
\hline IMID vs. Non-IMID & 0.97 & $0.93,1.00$ \\
Age (10-year increase) & 0.89 & $0.89,0.90$ \\
Sex: Female vs. Males & 0.95 & $0.93,0.97$ \\
LTC & 18.64 & $17.9,19.42$ \\
ADG score: & & \\
•5-9 vs. 0-4 & 1.40 & $1.35,1.45$ \\
•10-14 vs. 0-4 & 1.73 & $1.67,1.80$ \\
•15+ vs. 0-4 & 1.18 & $1.13,1.23$ \\
Urban vs. Rural & 3.49 & $3.26,3.72$ \\
Income quintile & & \\
•Quintile 2 vs. 1 & 0.92 & $0.89,0.96$ \\
•Quintile 3 vs. 1 & 0.90 & $0.86,0.93$ \\
•Quintile 4 vs. 1 & 0.73 & $0.70,0.76$ \\
•Quintile 5 vs. 1 & 0.60 & $0.58,0.63$
\end{tabular}

ADG - Aggregated Diagnosis Groups; IMIDs - Immune medicated inflammatory disease; LTC - long term care

Acknowledgements: The study is supported by ICES, which is funded by an annual grant from the Ontario Ministry of Health and Long-Term Care (MOHLTC). Parts of this material are based on data and information compiled and provided by MOHLTC and the Canadian Institute for Health Information. The opinions, results and conclusions reported in this paper are those of the authors and are independent of the funding or data sources; no endorsement is intended or should be inferred.

Disclosure of Interests: None declared.

DOI: 10.1136/annrheumdis-2021-eular.1647

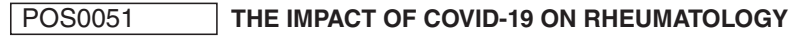 TRAINING: RESULTS FROM THE COVID-19 GLOBAL RHEUMATOLOGY ALLIANCE TRAINEE SURVEY}

K. Young ${ }^{1}$, S. A. Yeoh ${ }^{2}$, M. Putman ${ }^{3}$, E. Graef ${ }^{4}$, F. Berenbaum ${ }^{5,6,7}$, R. Conway ${ }^{8}$, R. Grainger ${ }^{9}$, A. Kilian ${ }^{10}$, M. Konig ${ }^{11}$, J. Liew ${ }^{12}$, P. M. Machado ${ }^{2}$, S. E. Sattui ${ }^{13}$, J. Sparks ${ }^{14,15}$, P. Sufka ${ }^{16}$, M. Ugarte-Gil ${ }^{17}$, L. Upton ${ }^{18}$, Z. Wallace ${ }^{15,19}$,

J. Yazdany ${ }^{20}$, A. Jayatilleke ${ }^{21}$ on behalf of the COVID-19 Global Rheumatology Alliance. ' University of Texas Southwest Medical Center, Rheumatology, Dallas, United States of America; ${ }^{2}$ University College London, Rheumatology, London, United Kingdom; ${ }^{3}$ Medical College of Wisconsin, Rheumatology, Milwaukee, United States of America; ${ }^{4}$ Emerson Hospital, Department of Medicine, Concord, United States of America; ${ }^{5}$ Sorbonne University, Rheumatology, Paris, France; ${ }^{6}$ AP-HP Saint-Antoine Hospital, Rheumatology, Paris, United Kingdom; ${ }^{7}$ Inserm, Rheumatology, Paris, France; ${ }^{8}$ St. James's Hospital, Rheumatology, Dublin, Ireland; ${ }^{9}$ University of Otago Wellington, Department of Medicine, Wellington, New Zealand; ${ }^{10}$ George Washington University, School of Medicine and Health Sciences, Washington DC, United States of America; ${ }^{11}$ Johns Hopkins University, Rheumatology, Baltimore, United States of America; ${ }^{12}$ Boston University School of Medicine, Section of Rheumatology, Boston, United States of America; ${ }^{13} \mathrm{Hospital}$ for Special Surgery, Department of Medicine, New York, United States of America; ${ }^{14}$ Brigham and Women's Hospital, Rheumatology, Boston, United States of America; ${ }^{15} \mathrm{Harvard}$ Medical School, Rheumatology, Boston, United States of America; ${ }^{16}$ HealthPartners \& Regions Hospital, Rheumatology, St. Paul, United States of America; ${ }^{17}$ Universidad Cientifica del Sur, Rheumatology, Lima, Peru; ${ }^{18}$ Georgetown University, School of Medicine, Washington DC, United States of America; ${ }^{19}$ Massachusetts General Hospital, Division of Rheumatology, Allergy, and Immunology, Boston, United States of America; ${ }^{20}$ University of California San Francisco, Division of Rheumatology, San Francisco, United States of America; ${ }^{21}$ Temple University Lewis Katz School of Medicine, Rheumatology, Philadelphia, United States of America 